\title{
ENSINO DE GRADUAÇÃO NA ESCOLA DE ENFERMAGEM DE RIBEIRÃO PRETO DA UNIVERSIDADE DE SÃO PAULO AO LONGO DOS SEUS 50 ANOS (1953-2003)
}

\author{
Maria José Clapis ${ }^{1}$ \\ Maria Suely Nogueira ${ }^{\top}$ \\ Débora Falleiros de Mello ${ }^{\top}$ \\ Adriana Kátia Corrêa \\ Maria Conceição Bernardo de Melo e Souza ${ }^{1}$ \\ Maria Manuela Rino Mendes ${ }^{1}$
}

Clapis MJ, Nogueira MS, Mello DF, Corrêa AK, Souza MCBM, Mendes MMR. O ensino de graduação na Escola de Enfermagem de Ribeirão Preto da Universidade de São Paulo ao longo dos seus 50 anos (1953-2003). Rev Latino-am Enfermagem 2004 janeiro-fevereiro; 12(1):7-13.

Este artigo tem por objetivo descrever a trajetória do ensino de graduação desenvolvido na Escola de Enfermagem de Ribeirão Preto da Universidade de São Paulo (EERP-USP), ao longo dos seus 50 anos (1953-2003), trazendo subsídios para reflexões acerca do contexto histórico-social, das diretrizes curriculares atuais e desafios político-pedagógicos para a formação do enfermeiro.

DESCRITORES: enfermagem; educação em enfermagem; programas de graduação em enfermagem; educação superior

\section{UNDERGRADUATE EDUCATION AT THE UNIVERSITY OF SÃO PAULO AT RIBEIRÃO PRETO COLLEGE OF NURSING THROUGHOUT THE 50 YEARS OF ITS HISTORY (1953-2003)}

This study aims to describe the course of undergraduate education developed at the University of São Paulo at Ribeirão Preto College of Nursing throughout the 50 years of its history (1953-2003), offering tools for reflection about the historical and social context, about current curricular guidelines and political and pedagogical challenges with a view to nursing formation.

DESCRIPTORS: nursing; education, nursing; undergraduate nursing programs; education, higher

\section{LA EDUCACIÓN DE PRE-GRADO EN LA ESCUELA DE ENFERMERÍA DE RIBEIRÃO PRETO DE LA UNIVERSIDAD DE SÃO PAULO A LO LARGO DE SUS 50 AÑOS (1953-2003)}

En este estudio, el objetivo es describir la trayectoria de la educación de pre-grado desarrollada en la Escuela de Enfermería de Ribeirão Preto de la Universidad de São Paulo a lo largo de sus 50 años (1953-2003), trayendo aportes para reflexionar a cerca del contexto histórico y social, de las directrices curriculares actuales y los desafíos políticos y pedagógicos para la formación del enfermero.

DESCRIPTORES: enfermería; educación en enfermería; programas de graduación en enfermería; educación superior

\footnotetext{
${ }^{1}$ Membros da Comissão de Graduação da Escola de Enfermagem de Ribeirão Preto, da Universidade de São Paulo, Centro Colaborador da OMS para o desenvolvimento da pesquisa em enfermagem, e-mail: gradenf@eerp.usp.br
} 
INTRODUÇÃO

Ao longo dos anos, a Escola de Enfermagem de Ribeirão Preto, através de suas atividades de ensino, pesquisa e extensão, vem procurando consolidar um projeto pedagógico que contemple a formação de um profissional, agente de mudanças, preocupado com os rumos da sociedade, bem como com o desenvolvimento de sua profissão. Desde o início de suas atividades, a Escola teve como filosofia desenvolver um curso de enfermagem adequado à realidade brasileira.

Deter o olhar na trajetória do ensino de graduação desta Escola nos estimula a refletir sobre nosso fazer político-pedagógico e as perspectivas e desafios a serem enfrentados no atual contexto de educação e saúde.

\section{O ENSINO DE GRADUAÇÃO NA EERP-USP: CONTEXTO HISTÓRICO-SOCIAL}

A EERP/USP foi criada em 1951, anexa à Faculdade de Medicina de Ribeirão Preto-USP e o curso foi iniciado em 1953, tendo como fundadora a Prof ${ }^{a} \mathrm{Dr}^{\mathrm{a}}$ Glete de Alcântara. A fundação e organização da Escola tiveram a relevante contribuição dessa professora, formada pela School of Nursing of Toronto, Canadá, em 1944, tendo cursado Ciências Sociais na Universidade de São Paulo e Mestrado no Teacher's College, University of Columbia, em $1951^{(1)}$.

A Prof ${ }^{a}$ Dra Glete de Alcântara foi diretora da EERP/USP por 20 anos. Possuía todas as qualidades e, ao mesmo tempo, vigor para um projeto acadêmico, possibilitando à Escola nascente um caráter inovador, aberto e adequado à realidade da época. A ênfase desse projeto estava voltada para o encontro de respostas às necessidades de saúde da comunidade, com o estabelecimento de estrutura didático-administrativa para formação de profissionais na prestação de assistência e direção de unidades hospitalares e de centros de saúde. Foi criada uma Escola sem internato obrigatório, até então registro indispensável nas instituições destinadas a formar enfermeiros, sendo a primeira escola a aceitar alunos externos, mantendo internato para aqueles que residissem fora de Ribeirão Preto ${ }^{(1)}$.

Nos anos 50 e 60, o marco conceitual do ensino incluía a integração de aspectos sociais e psicológicos para a assistência de enfermagem e aspectos preventivos e curativos na saúde, além do enfoque de administração de serviços. A enfermagem, no Brasil, nesse período, passou por uma evolução que era fruto da ampliação do parque industrial, do crescimento da população urbana e previdenciária e, ao mesmo tempo, de um aumento crescente de escolas de enfermagem e de cursos de auxiliar de enfermagem. Em 1955, foi criada a Lei do Exercício Profissional oㅡ 2604 para reger a profissão ${ }^{(2)}$.

Em 1964, a EERP foi desanexada da Faculdade de Medicina de Ribeirão Preto, tornando-se estabelecimento de nível superior. No período de 1953 a 1966, o curso de enfermagem geral tinha a duração de 4 anos, situação que se mantém até o presente momento. Em 1967, a Escola passou a oferecer o curso de enfermagem geral em 3 anos e mais um ano opcional, com curso de enfermagem em saúde pública. Em 1969, no $4^{\circ}$ ano de graduação, havia a possibilidade de opção em enfermagem médico-cirúrgica, enfermagem psiquiátrica e enfermagem pediátrica. A partir de 1975, com base no Parecer 163/72 do Conselho Federal de Educação, passaram a ter vigência as habilitações em enfermagem médico-cirúrgica, enfermagem em saúde pública e enfermagem obstétrica. Para o ingresso na EERP/USP, desde a sua criação até o ano de 1967, era necessário que o candidato possuísse o curso ginasial completo ou equivalente e fosse aprovado em exame vestibular, realizado pela própria escola, nas disciplinas de física, biologia e química. No período de 1968 a 1976, a condição de ingresso estava vinculada à conclusão do curso colegial ou equivalente, e aprovação no vestibular pelo Centro de Seleção de Candidatos às Escolas (CESEM), entidade responsável pelo processo seletivo a aspirantes da área biológica nas Escolas Públicas do Estado de São Paulo, cujas provas eram realizadas em uma única fase, com utilização de testes de múltipla escolha. A partir de 1977, a seleção para ingresso no curso passou a ser de responsabilidade da Fundação Universitária para o Vestibular (FUVEST), realizado em duas fases, sendo a primeira com questões de múltipla escolha e a segunda, com questões dissertativas. Da mesma forma exige-se como requisito a conclusão do $2^{\circ}$ grau ou equivalente. No tocante ao número de vagas, no período de 1953 a 1960, era 30, entre 1961 e 1965 foi diminuído para 20, de 1966 a 1975 foi aumentado para 40 e, a partir de 1976, passou para 80 vagas, número que permanece até hoje. Quanto ao certificado de conclusão do curso recebido pelos alunos, no período de 1953 a 
1968, era o Diploma de Enfermeiro, entre 1969 e 1975, era o de Enfermeiro e o de Enfermeiro de Saúde Pública, a partir de 1976, o Diploma de Bacharel em Enfermagem e o correspondente à habilitação escolhida, sendo a habilitação em enfermagem obstétrica oferecida até $1979^{(3)}$.

Na década de 70, a Escola passou a incorporar algumas modificações em sua estrutura acadêmica e administrativa, instalando os Departamentos e a Congregação, instituindo o sistema de créditos nas disciplinas, matrículas semestrais, vestibular unificado e regulamentando a Comissão de Ensino como órgão assessor da Direção e responsável pela coordenação didática, assim como o início dos cursos de pós-graduação e especialização.

A estrutura curricular da EERP/USP, na década de 70, aprovada em 1974, refletia mudanças no ensino de graduação com a inclusão de uma série de disciplinas para atender às exigências mínimas da formação do enfermeiro, conforme Parecer no $163 / 72$, com ênfase para o mercado de trabalho, centrado, predominantemente, no contexto hospitalar.

Nos anos 80, a Escola realizou um estudo sobre reforma curricular, com discussões sobre a necessidade do ensino estar voltado para as políticas de extensão de cobertura e de atenção primária em saúde, sobre a concepção teórica de um currículo, partindo-se do princípio de que o currículo, mais do que um rol de disciplinas, é a seqüência de experiências através das quais a Escola tenta estimular o desenvolvimento do aluno, proporcionando conhecimento pessoal para o aprendiz nas dimensões intelectual, afetiva, motora e social. A proposta de reformulação estava voltada para a formação do enfermeiro generalista, centrando o ensino nas competências das diversas áreas de atuação profissional ${ }^{(2)}$.

O eixo condutor desse novo currículo era o atendimento hierárquico em níveis de complexidade, partindo do ensino teórico-prático em unidades básicas de saúde, para unidades de referência secundária e terciária ${ }^{(4)}$.

Após ampla discussão, envolvendo todos os segmentos da Escola, em 1989, implanta-se o currículo para formação do enfermeiro generalista, com estrutura curricular composta de um ciclo pré-profissional e profissional. O primeiro compreendendo os princípios gerais básicos de um currículo para a área da saúde, com os núcleos de ciências do comportamento, da biologia, da epidemiologia e da educação em saúde, e o segundo voltado a capacitar o aluno para o desenvolvimento da assistência individual e coletiva dirigida à criança, mulher e adulto, nas áreas de enfermagem clínica, cirúrgica, obstétrica, ginecológica, pediátrica e psiquiátrica, ficando o último semestre do curso para capacitação em administração dos serviços básicos de saúde e da área hospitalar.

O currículo de 1989, com carga horária total de 4095 horas, tinha abordagem multidisciplinar, com a proposta de levar o aluno a uma visão global, integrada e crítica da profissão e da atuação profissional, buscando competência técnico-científica, administrativa e política do enfermeiro para atuar nos diferentes serviços de saúde e em níveis de complexidade distintos, evidenciando também a relevância da pesquisa para o desenvolvimento da profissão ${ }^{(2)}$.

É importante ressaltar que a Comissão de Graduação manteve um grupo de docentes assessores que avaliavam periodicamente o andamento e os resultados da renovação curricular, através da realização de reuniões entre alunos e professores dos diversos semestres ${ }^{(4)}$. A partir dessas avaliações, algumas adequações de carga horária e alterações de disciplinas na grade curricular foram efetuadas, mas que não modificaram o projeto pedagógico em si.

Em 1998, nova reformulação foi realizada para a implantação da disciplina Estágio Curricular em Enfermagem, entendida como um procedimento didático que conduz o aluno a situar, observar e aplicar, criteriosa e reflexivamente, princípios referenciais teórico-práticos assimilados no decorrer do curso. A disciplina estágio curricular tem como objetivo proporcionar aos alunos experiências acadêmico-profissionais, com reflexões ativas e críticas no ambiente de trabalho do enfermeiro, favorecendo uma dimensão socioeducacional e, seu caráter profissionalizante permitindo ao aluno a percepção do compromisso social que deve permear a futura atuação profissional $^{(3)}$.

Analisando o processo de reestruturação curricular de 1998 (Proc. USP no 97.1.432.22.7), observamos que as modificações propostas objetivaram proporcionar um espaço na grade curricular para a criação das disciplinas de Estágio Curricular no último semestre do Curso de Graduação em Enfermagem. Assim, a reformulação tratou, basicamente, da reorganização das disciplinas com diminuição do número de créditos, como 
possibilidade de otimização do tempo, com remodelação da metodologia de ensino e manutenção da qualidade do processo ensino-aprendizagem, garantindo o conteúdo básico ${ }^{(3)}$

Com esse projeto pedagógico, a EERP/USP propõe-se a preparar um profissional crítico e reflexivo, com competência técnico-científica e ético-política, responsável pelo cuidado de enfermagem e pelo processo de formação dos trabalhadores de enfermagem, que respeita os princípios éticos e legais da profissão, valorizando o ser humano em sua totalidade e o exercício da cidadania, no resgate da qualidade de vida, comprometido com sua qualificação permanente e com o desenvolvimento da profissão. O profissional formado por essa instituição deve ser capaz de:

- prestar o cuidado de enfermagem, identificando as necessidades individuais e coletivas da população e seus determinantes, intervindo no processo saúde-doença com vistas à qualidade da assistência de enfermagem em todos os níveis de atenção à saúde;

- gerenciar a assistência de enfermagem, tomando decisões nos diferentes níveis de atenção à saúde;

- desenvolver e participar de pesquisas bem como implementar os resultados das mesmas com vistas à evolução da prática de enfermagem e de saúde;

- planejar e implementar ações de educação em saúde dirigidas à população;

- avaliar continuamente os resultados e o impacto das ações desenvolvidas;

- trabalhar articulado com profissionais de outras áreas da saúde e sociedade ${ }^{(3)}$.

Para viabilizar o perfil apresentado, deve-se investir no processo de formação, em competências e habilidades que permitam ações assistenciais (preventivas, curativas e de reabilitação), educativas, gerenciais e de investigação em saúde.

Cabe ressaltar que em 1994 a Escola criou o Curso de Licenciatura em Enfermagem, oferecido na própria instituição, visando preparar o enfermeiro para o ensino básico formal (fundamental e médio) para lecionar junto aos programas de Higiene e Saúde, bem como para o ensino médio profissionalizante em enfermagem. $O$ curso de Licenciatura em Enfermagem trata-se de um espectro optativo para o processo de formação extensiva do curso de graduação.

Esse curso, frente às mudanças políticas e legais da formação de profissionais no contexto da educação brasileira e da própria Universidade, está, no momento, passando por processo de análise e avaliação.

As reformas educacionais instituídas no Brasil, desde a promulgação da Lei de Diretrizes e Bases da Educação Nacional (LDB de 20/12/1996), entre outros aspectos, vêm determinando novas configurações aos padrões curriculares que, até recentemente, vigoravam em todos os níveis de modalidade de ensino ${ }^{(5)}$.

A promulgação de diretrizes curriculares para os cursos de formação profissional, em nível de graduação, está inserida em um discurso que anuncia ampla reforma educacional do ensino superior brasileiro e coloca verdadeiros desafios para as instituições de ensino na revisão de seus Projetos Pedagógicos.

\section{DIRETRIZES POLÍTICO-PEDAGÓGICAS: LIMITES E DESAFIOS}

As diretrizes curriculares constituem um conjunto de indicações que devem direcionar o processo educacional do ensino superior, orientando o planejamento acadêmico dos cursos de graduação. Caracterizam-se pela flexibilidade, buscando romper com o modelo arcaico e rígido de ensino, trazendo o Projeto Pedagógico como base de gestão acadêmico-administrativa de cada curso e fornecendo os elementos das bases filosóficas, conceituais, políticas e metodológicas que definem as competências e habilidades essenciais à formação dos profissionais de saúde ${ }^{(6)}$.

Nos cursos da área da saúde, as diretrizes curriculares estão orientadas para contribuir no processo de construção do Sistema Único de Saúde (SUS), o que vem ao encontro da Constituição Federal que indica o papel relevante do SUS no delineamento de diretrizes para a formação de recursos humanos em saúde.

Considerando os princípios orientadores do SUS, dentre eles, a eqüidade, a integralidade da atenção, a participação social do usuário, fundamentados na compreensão do processo saúde-doença para além do eixo técnico-biológico, na perspectiva de construção da cidadania, faz-se cada vez mais necessária a reformulação do processo formativo dos profissionais de saúde, necessidade essa expressa nas diretrizes curriculares dos cursos de saúde, através da construção de novos perfis e competências profissionais.

Nesse contexto, os movimentos pela reforma 
sanitária brasileira e pela mudança na formação dos profissionais de saúde propõem alguns objetivos que têm sido amplamente discutidos: atenção à saúde universal, eqüitativa e de qualidade com enfoque na promoção da saúde e prevenção das doenças; universalidade voltada à produção de conhecimento que seja relevante socialmente e útil para a construção do SUS; profissional crítico, com competência para aprender a aprender e trabalhar em equipe, considerando a realidade social, para garantir um atendimento humano e de qualidade ${ }^{(7)}$. A autora comenta, ainda, que os alcances desses objetivos, nos campos da educação e da saúde exigem mudanças, pois, mesmo considerando os avanços já obtidos na construção do SUS, a transformação do modelo de atenção se encontra na esfera do desejo, como um avanço que não foi completamente concretizado.

Do mesmo modo, consideramos que os novos perfis e competências profissionais, no contexto desta Escola, estão também nessa "esfera do desejo", demandando transformações na ação político-pedagógica. Transformações essas que se inserem, inclusive, em um processo mais amplo de necessidade de mudanças no contexto educacional, em âmbito mundial, com vistas à construção de relações sociais mais justas e éticas.

Nas últimas décadas, a velocidade e diversidade das mudanças sociais têm gerado inovações nas políticas governamentais, voltadas para o sistema educacional, entendido como elemento chave para desencadear o desenvolvimento humano e o progresso social, evidenciado pela eqüidade de acesso a bens disponíveis ${ }^{(8)}$.

Expressão desse movimento pode ser observada nas novas políticas da educação, formulando as diretrizes para formação humana no país, nos níveis de ensino básico, médio e superior. O grande desafio está em incorporar o modo ativo e responsável de:

- aprender a conhecer - distingüindo o real do ilusório, estabelecendo relações entre os diferentes saberes e significados na vida cotidiana, cuja abordagem transdisciplinar possibilite adaptações às mudanças da vida profissional;

- aprender a fazer - representado pela aquisição de conhecimentos e práticas associadas a uma profissão, edificando um núcleo flexível capaz de permitir o aprendizado com criatividade;

- aprender a viver juntos - respeitando regulamentos de relações entre seres que compõem o coletivo, compreendendo, admitindo e tolerando as diferenças, reconhecendo-se nos outros e fortalecendo convicções e posições;

- aprender a ser - descobrindo-se como indivíduo e parte da sociedade, formulando questões fundamentais, desenvolvendo o espírito científico ${ }^{(8)}$.

Esse desafio vai exigir mudanças no sistema educacional, nas instituições, nas relações com outros setores e entre os atores, envolvendo a construção de novos papéis para professores e alunos, considerando também a tendência das sociedades da informação e do conhecimento. Diante do exposto, apreende-se que o processo formativo de enfermeiros desta Escola deve contemplar um projeto educacional que realmente contribua para a formação de cidadãos que possam se inserir de modo crítico e responsável no contexto sociocultural, político e econômico da realidade brasileira.

Apesar do projeto político-pedagógico atual de nossa Escola visar a formação de um profissional crítico e reflexivo, com competência técnico-científica e éticopolítica, a concretização desse "ideal" no cotidiano do ensino é questionável. Ou seja, a construção de nosso(s) projeto(s) pedagógico(s) nos espaços de ensinoaprendizagem, envolvendo as relações entre docentes, alunos e profissionais, os saberes instituídos, bem como nossos posicionamentos políticos e éticos no contexto da saúde e educação, muitas vezes, se processa de maneira que não condiz com a formação almejada.

Nesse contexto, vem se mostrando urgente a necessidade de criar um espaço coletivo de diálogo que promova a discussão de nosso fazer político-pedagógico, envolvendo distintos atores, tomando como eixo os parâmetros presentes nas atuais diretrizes curriculares: projeto pedagógico construído coletivamente, flexibilidade, formação integral, incorporação de atividades complementares em relação ao eixo fundamental do processo de formação, interdisciplinaridade, predominância da formação sobre a informação, articulação entre teoria e prática e indissociabilidade entre ensino, pesquisa e extensão(6).

Uma das questões que deve nortear nossas reflexões é o quanto estamos próximos ou afastados da concepção de projeto político-pedagógico que desejamos, cabendo ressaltar que os docentes que, no momento, compõem a Comissão de Graduação, vêm empreendendo esforços na busca de viabilizar essas reflexões, envolvendo a comunidade escolar. Esses esforços estão sendo motivados por alguns aspectos como as mudanças 
necessárias na formação do enfermeiro, tendo em vista o contexto atual de saúde e educação, as discussões que ocorrem nos cenários formais e informais da escola e de instituições de serviços de saúde, envolvendo discentes, professores e profissionais, revelando insatisfações com a formação, as avaliações das disciplinas realizadas pelos alunos, nos distintos semestres, mostrando o predomínio do ensino tradicional que se encontra na contramão dos princípios que devem nortear os projetos políticopedagógicos da área da saúde como, por exemplo, a utilização de metodologias ativas do processo ensinoaprendizagem, a integração de conteúdos básicos e profissionalizantes, a educação voltada aos problemas mais relevantes da sociedade, a avaliação formativa, entre outros $^{(9)}$.

Todavia, compor espaços para discussão coletiva vem se mostrando uma tarefa árdua, sendo apontadas algumas dificuldades, pelos docentes, em reuniões já realizadas, com o propósito de iniciar um processo reflexivo sobre nosso projeto político-pedagógico, em 2003. A Comissão de Graduação tem coordenado reuniões onde depreende-se alguns aspectos dificultadores: a estrutura organizacional departamentalizada, o número elevado de discentes, a incipiente formação pedagógica, a desarticulação com os serviços de saúde, a organização do trabalho docente e a sua valorização, principalmente, pelo desempenho na produção de pesquisas, com pouca ênfase nas atividades de graduação e as dificuldades inerentes à realização de mudanças individuais e coletivas no cotidiano de ensino ${ }^{(10)}$.

Pensamos que essas dificuldades precisam ser levadas em conta, pois elas nos dão, de certo modo, o tom daquilo que é possível, nesse momento, nesse contexto, porém não as tomamos como imobilizadoras de nossa ação crítico-criativa.

E, nesse contexto, compreendemos que a noção de projeto implica sobretudo em tempo:

- tempo político - refere-se à oportunidade política de dado projeto;

- tempo institucional - cada escola encontra-se em tempo determinado de sua história. Assim, um projeto pode ser inovador para uma escola e não o ser para outra;

- tempo escolar - relaciona-se ao calendário escolar. $O$ período no qual o projeto é elaborado é também decisivo para seu sucesso;

- tempo para amadurecer as idéias - projetos burocráticos, por serem impostos, mostram-se ineficientes a médio prazo. É necessário tempo para sedimentar idéias. Um projeto necessita ser discutido e isso realmente leva tempo ${ }^{(11)}$.

A formação de recursos humanos em saúde tem, atualmente, como focos centrais os esforços para articular elementos do campo da educação e do trabalho, enfatizar a Lei de Diretrizes e Bases da Educação como fundamental para reconhecer a necessidade de construção de novos modelos de ensino e enfocar a competência como estruturadora da base curricular ${ }^{(12)}$.

A ação educativa é um processo complexo que pode servir à dominação ou à libertação dos indivíduos. $O$ espaço da dominação está ligado à formação de sujeitos dependentes, não críticos, acomodados às informações que são transmitidas e facilmente manipuláveis. O espaço denominado libertador envolve a formação de indivíduos críticos, independentes, com capacidade de reflexão sobre a realidade nos âmbitos educacional, político, econômico e cultural ${ }^{(13)}$

Para êxito de um projeto pedagógico são considerados como elementos facilitadores à comunicação, as adesões voluntárias e conscientes ao projeto, envolvendo co-responsabilidade, suporte institucional, imbuído de vontade política e recursos financeiros claramente definidos, controle, acompanhamento e avaliação do projeto, credibilidade, referenciais teóricos, possibilitando conceituações e estruturação do projeto, e atmosfera favorável, configurando um componente mágico-simbólico para o sucesso do projeto $^{(9)}$.

Assim, é de extrema importância a fundamentação nesses conceitos e discussões para avançarmos no nosso processo educativo.

\section{CONSIDERAÇÕES FINAIS}

A construção do projeto pedagógico de um curso de graduação deve considerar suas especificidades institucionais e locais, contemplando a análise de seus aspectos histórico-sociais, seus limites, desejos e desafios, que procuramos refletir no presente artigo.

As novas práticas de formação dos profissionais de saúde necessitam estar sendo constantemente repensadas, buscando o enfrentamento de nossas contradições e ampliando nossa compreensão sobre as temáticas: competência, qualidade no ensino, educação 
voltada às demandas do mercado de trabalho/formação integral, interdisciplinaridade, saberes essenciais, formação generalista, entre outras.

O debate atual vem colocando em pauta a política de educação para o SUS, a importância da interação entre os segmentos ensino-serviço-gestão setorial-controle social em saúde e a formação e desenvolvimento dos profissionais de saúde, integrando setores saúde e educação. Dessa forma, compreendemos que a enfermagem está inserida nesse processo, buscando avançar na formação profissional, construindo e refletindo o projeto de nossa comunidade escolar.

\section{REFERÊNCIAS BIBLIOGRÁFICAS}

1. Angerami ELS, Pelá NTR. Glete de Alcântara: vida e obra. São Paulo (SP): Revista dos Tribunais; 1976.

2. Freitas DMV, Fávero N, Scatena MCM. O ensino de graduação na Escola de Enfermagem de Ribeirão Preto da USP: suas perspectivas. Rev Latino-am Enfermagem 1993 dezembro; 1 (no especial):25-34.

3. Universidade de São Paulo. Escola de Enfermagem de Ribeirão Preto (BR). Projeto Pedagógico: curso de enfermagem. Ribeirão Preto (SP): EERP/USP; 2001.

4. Angerami ELS. Relatório de gestão - 1986-1990. Ribeirão Preto (SP): Escola de Enfermagem de Ribeirão Preto/USP; 1990.

5. Grossi E. LDB: Lei de Diretrizes e Bases da Educação Lei no 9.394/96. Rio de Janeiro (RJ): DP\&A Editora; 1998.

6. Xavier IM, Fernandes JD, Ceribelli MIPF. Diretrizes curriculares: articulação do texto e contexto - breve comentário. Bol Inf Assoc Bras Enfermagem 2002 setembro; 44(2):6-7.

7. Feuerwerker L. Estratégias de mudança da formação dos profissionais. Olho Mágico. 2002; 9(1):16-8.

8. Delor's J, organizador. Educação: um tesouro a descobrir - Relatório Unesco da Comissão Internacional sobre Educação para o século XXI. 6ªed. Brasília (DF): UNESCO/ MEC/Cortez; 2001.

9. Gadotti M, Yamazaki AA, Gomes AMV, Ciseski AA, Mânfio AJ, Maldonado CA, et al. Perspectivas atuais da educação. Porto Alegre (RS): Artmed; 2000.

10. Universidade de São Paulo. Escola de Enfermagem de Ribeirão Preto. Comissão de Graduação (BR). Síntese da reunião para discussão do atual projeto político-pedagógico da escola - dia 31/03/2003. Ribeirão Preto (SP): EERP/USP; 2003.

11. Universidade de São Paulo. Escola de Enfermagem de Ribeirão Preto. Comissão de Graduação (BR). Síntese da reunião para discussão do atual projeto político-pedagógico da escola - dia 11/04/2003. Ribeirão Preto (SP): EERP/USP; 2003.

12. Rede Unida: Contribuição para as novas diretrizes curriculares dos cursos de graduação da área da saúde. Olho Mágico 1999; 5(nำespecial).

13. Sordi MRL, Bagnato, MHS. Subsídios para uma formação crítico-reflexiva na área de saúde: o desafio da virada do século. Rev Latino-am Enfermagem 1998 abril; 6(2):83-8. 